\title{
Cutaneous Leishmaniasis in dogs: is high seroprevalence indicative of a reservoir role?
}

\author{
JOSÉ E. CALZADA ${ }^{1,2}$, AZAEL SALDAÑA ${ }^{1}$, KADIR GONZÁLEZ ${ }^{1}$, CHYSTRIE RIGG ${ }^{1}$, \\ VANESSA PINEDA ${ }^{1}$, ANA MARÍA SANTAMARÍA ${ }^{1}$, INDRA RODRÍGUEZ ${ }^{2}$, \\ NICOLE L. GOTTDENKER ${ }^{3}$, MARCIA D. LAURENTI ${ }^{4}$ and LUIS F. CHAVES ${ }^{5,6 *}$ \\ ${ }^{1}$ Departamento de Parasitología, Instituto Conmemorativo Gorgas de Estudios de Salud (ICGES), Ciudad de Panamá, \\ República de Panamá \\ ${ }^{2}$ Facultad de Medicina Veterinaria, Universidad de Panamá, Ciudad de Panamá, República de Panamá \\ ${ }^{3}$ Department of Veterinary Pathology, College of Veterinary Medicine, University of Georgia, Athens, Georgia, USA \\ ${ }^{4}$ Departamento de Patología, Facultade de Medicina, Universidade de São Paulo, São Paulo, Brasil \\ ${ }^{5}$ Institute of Tropical Medicine (NEKKEN), Nagasaki University, 852-8523 Sakamoto 1-12-4, Nagasaki, Japan \\ ${ }^{6}$ Programa de Investigación en Enfermedades Tropicales (PIET), Escuela de Medicina Veterinaria, Universidad Nacional, \\ Heredia, Costa Rica
}

(Received 14 November 2014; revised 11 March 2015; accepted 6 April 2015; first published online 20 May 2015)

\section{SUMMARY}

American cutaneous leishmaniasis (ACL) is a complex disease with a rich diversity of animal host species. This diversity imposes a challenge, since understanding ACL transmission requires the adequate identification of reservoir hosts, those species able to be a source of additional infections. In this study we present results from an ACL cross-sectional serological survey of 51 dogs (Canis familiaris), where we used diagnostic tests that measure dog's exposure to Leishmania spp. parasites. We did our research in Panamá, at a village that has undergone significant ecosystem level transformations. We found an ACL seroprevalence of $47 \%$ among dogs, and their exposure was positively associated with dog age and abundance of sand fly vectors in the houses of dog owners. Using mathematical models, which were fitted to data on the proportion of positive tests as function of dog age, we estimated a basic reproductive number $\left(R_{0} \pm\right.$ S.E. $)$ of $1 \cdot 22 \pm 0 \cdot 09$ that indicates the disease is endemically established in the dogs. Nevertheless, this information by itself is insufficient to incriminate dogs as ACL reservoirs, given the inability to find parasites (or their DNA) in seropositive dogs and previously reported failures to experimentally infect vectors feeding on dogs with ACL parasites.

Key words: Canis familiaris, Leishmania panamensis, reservoirs, endemicity, force of infection, catalytic models.

\section{INTRODUCTION}

American cutaneous leishmaniasis (ACL) is a complex disease system, with several interacting socio-ecological factors underpinning its transmission dynamics (Chaves et al. 2008a). Along the social axis of its complexity, over the years, it has become clear that ACL is a disease intimately related with patterns of inequity in wealth distribution and poverty (Alvar et al. 2012). Along the ecological axis of its complexity, ACL is a disease system that serves as a model to study major ecological processes and biodiversity patterns, beginning with a rich diversity of vectors, animal host species (Christensen and de Vasquez, 1982; Christensen et al. 1983; Dutari and Loaiza, 2014) and parasites whose interactions change with ecosystem-wide disturbances (or irreversible shifts), such as deforestation and biodiversity destruction (Alexander et al. 2001; Quintana et al. 2010). When social and ecological axes converge, ACL emerges as a disease with a large diversity of epidemiological patterns, where, for example, poor housing quality may impair vector control success

* Corresponding author. Institute of Tropical Medicine (NEKKEN), Nagasaki University, 852-8523 Sakamoto 1-12-4, Nagasaki, Japan. E-mail: lchaves@nagasaki-u.ac.jp
(Chaves et al.2013), and the identification of major vertebrate reservoir hosts becomes a major challenge in settings where domestic animals and wildlife species coexist (Chaves et al. 2007).

Reservoirs are mammal hosts with dynamic feedbacks in pathogen transmission, i.e. animals able to serve as source of pathogens to vectors and/or new hosts (Viana et al. 2014). In the case of ACL this equates to mammal species that are a common source of infection for sand flies, something possible when a given mammal species: (i) is commonly bitten by sand flies that also bite humans, (ii) has life history and immunological traits that allow Leishmania spp. parasites development and transmission after being infected, (iii) and where parasites can be easily isolated from blood or tissues where sand flies can get infected. Interestingly, ACL reservoir hosts do not necessarily present ACL associated pathologies and the infection does not need to be endemically established in their populations (Ashford, 1997; Chaves et al. 2007).

Reservoirs are particularly difficult to identify in ACL given the richness of recorded hosts for New World Cutaneous (NWC) Leishmania spp. parasites and the catholic blood feeding patterns of dominant vector species (Christensen et al. 1983; Dutari and

Parasitology (2015), 142, 1202-1214. C Cambridge University Press 2015. This is an Open Access article, distributed under the terms of the Creative Commons Attribution licence (http://creativecommons.org/licenses/by/3.0/), which permits unrestricted re-use, distribution, and reproduction in any medium, provided the original work is properly cited. 
Loaiza, 2014). Among ACL vertebrate host species, the domestic dog Canis familiaris has been the centre of controversy and there is a lack of consensus regarding its role as reservoir of NWC Leishmania spp. parasites (Falqueto et al. 1991; Reithinger and Davies, 1999; Dantas-Torres, 2007). From the initial report of $\mathrm{dog}$ infections in an endemic rural focus of ACL (Pedroso, 1913) and the suspicion of their role as likely reservoirs in the first urban ACL epidemic reported in the New World (Aragão, 1927), the high seroprevalence and the association between prevalence patterns in humans and dogs have been taken as major indicatives of a reservoir role for dogs in ACL epidemiology (Falqueto et al. 1991; Reithinger et al. 2003b). Nevertheless, studies considering Leishmania infections in other domestic animals have shown that equids, i.e. horses, donkeys and mules, were the likely reservoir in systems where dogs were originally suspected as NWC Leishmania reservoirs (Aguilar et al. 1989) a fact further reinforced by the low success probability of sand fly vectors at getting infected when biting dogs with NWC Leishmania spp. (Herrer and Christensen, 1976b; Hernández et al. 2006; Travi et al. 2006) and by mathematical modelling based on epidemiological reports where data on potential reservoir hosts including dogs and other species were collected (Chaves et al. 2007, 2008b).

In Panamá the main parasite currently causing ACL in humans is Leishmania panamensis (Chaves et al. 2014) and extensive research at the Gorgas Memorial Research Laboratory (current ICGES) in the 1960-1980s period showed the two toed sloth, Choloepus hoffmani, to be the main reservoir for L. panamensis (Christensen and Herrer, 1979; Herrer and Christensen, 1980). Furthermore, several studies suggested that dogs unlikely are ACL reservoirs in Panamá (Christensen and Herrer, 1973; Herrer and Christensen, 1976a, b). Similarly, to the best of our knowledge, the only other instance where ACL was studied in dogs with $L$. panamensis as the only circulating parasite was in the coast of Ecuador, and it did not find support for dogs as reservoirs (Dereure et al. 1994). Nevertheless, in Panamá, recent ACL epidemics in rural settings with a relatively recent history of major environmental change and urbanization (Saldaña et al. 2013) have raised questions about possible changes in the epidemiology of ACL. An outstanding question is whether dogs are becoming dominant reservoir hosts driving the dynamics of ACL transmission (Dantas-Torres, 2007). Here, we present results from a cross-sectional study in dogs from Trinidad de Las Minas, an endemic ACL rural community in Panamá Province, República de Panamá. We asked whether dogs were suitable reservoirs for ACL parasites, by attempting to detect ACL parasites and looking at their ACL seroprevalence patterns. We also studied the potential risk factors associated with ACL seropositive dogs. Briefly, we evaluated exposure to NWC Leishmania spp. in a population of 51 dogs by an indirect immunofluorescence assay test (IFAT) and an enzyme-linked immunosorbent assay (ELISA) using L. panamensis isolates as antigens. We also diagnosed active infections by parasite culture and polymerase chain reaction (PCR) tests from blood samples. Given the absence of a suitable gold standard (Reithinger et al. 2000), reflected in our study by the absence of CL lesions, negative PCR and parasite isolation results, as well as, the lack of agreement between the serological tests; we employed Bayesian methods to estimate the sensitivity and specificity of the serological tests (Branscum et al. 2005). We then evaluated risks factors for canine ACL seropositive reactions. Given that $R_{0}$, the basic reproduction number, which is defined as the expected number of new infections in a susceptible host population following the introduction of an infected host (Anderson and May, 1991), has been reified as a criterion to infer that dogs are ACL reservoirs when larger than one, i.e. when $R_{0}>1$ (Reithinger et al. 2003a), we employed maximum likelihood statistical methods to estimate the force of infection and $R_{0}$ from an age specific seroprevalence curve, to better illustrate how this parameter should be interpreted.

\section{METHODS}

\section{Study site}

We studied canine cutaneous leishmaniasis exposure in dogs from Trinidad de Las Minas ( $8^{\circ} 46^{\prime} 32^{\prime \prime} \mathrm{N}$; 7959'45" W), Panamá province, República de Panamá. Climate in the area is unimodal, with rainy (April-November) and dry (December-March) seasons. Rainfall ranges from 28 to $570 \mathrm{~mm}^{3}$ per month. Temperature is nearly constant with a yearround $26{ }^{\circ} \mathrm{C}$ average. Over recent years the native vegetation of our study site has been destroyed mainly for agricultural development, and the forest has become patchy and transitional (Chaves et al. 2013). We performed a census of dogs in May 2010, counting and recording individual data from all dogs in 24 households. We surveyed 24 houses; out of 198 in the village, because our resources were limited, especially the number of light traps available for Sand Fly (SF) sampling. Nevertheless, all houses enrolled in the study had confirmed SF presence (by the residents), had similar eco-epidemiological conditions and householders provided written informed consent to take samples from the animals. We also want to mention that assuming two dogs per house (Herrer and Christensen, 1976a,b), i.e. an approximate dog population size of 396 dogs in the whole village, a sample size of 48 dogs is large enough to detect a seroprevalence of at least $15 \%$ with a $10 \%$ 
precision, i.e. with $95 \%$ confidence intervals ranging from 5 to $25 \%$ (Sokal and Rohlf, 1994), prevalence values that have been previously recorded in Panamá (Herrer and Christensen, 1976a, b) and elsewhere canine cutaneous leishmaniasis is endemic in the New World (Reithinger and Davies, 1999).

\section{Dog sampling}

Households from the studied area were individually visited. Every dog in each household was physically examined with emphasis on the dog's skin and mucosa. Blood samples were collected from the cephalic vein under minimal stress with the consent and in the presence of the owners. Age, sex and body condition of each dog were assessed during sample collection following standard procedures described by Fung et al. (2014). Dog age was calculated based on the owner information, and independently corroborated by dentition and tartar deposition patterns. The presence of ectoparasites (such as fleas and ticks) was visually assessed with the help of a handheld magnifying lens. Blood samples were used for parasite isolation and for serological/molecular ACL diagnosis. Also, if skin lesions compatible with ACL were observed, scrapings were collected and spotted onto filter paper for molecular diagnosis. All dogs were dewormed after sampling.

\section{Parasite isolation and molecular analysis}

Blood samples were inoculated into biphasic Senekjie's medium with M199 (Sigma, St Louis, MO, USA) to attempt parasite isolation. Culture tubes were incubated at $26{ }^{\circ} \mathrm{C}$ and checked for Leishmania spp. promastigote presence every 7 days during 28 days. DNA was extracted from whole blood using the DNeasy extraction kit (Qiagen, Valencia, CA) according to the manufacturer's protocols. Primers targeting the entire 750bp minicircle of Leishmania Viannia sp. (Vergel et al. 2005) and the heat-shock protein 70 of New World Leishmania (Garcia et al. 2004) were used for PCR diagnosis. Amplification reactions were performed in a final volume of $50 \mu \mathrm{L}$. Internal positive and negative controls were used during each PCR analysis. Positive controls consisted of isolates from humans from the area (Miranda et al. 2012). A blood sample of a seronegative healthy dog from Ciudad de Panamá (non-endemic to Leishmania) was included in every PCR run as a negative extraction control. In every PCR we also included one reaction which contained only the reagents and no template, as a negative PCR control.

\section{ACL serology in dogs}

The presence of IgG antibodies against L. panamensis was assessed by ELISA and IFAT. For the ELISA, a total crude antigen derived from L. panamensis promastigotes (MHOM/PA/98/WR/ 2306) was implemented following the exact same steps described by Lemos et al. (2008) and Colombo et al. (2011). The detection of antibodies to L. panamensis by IFAT was carried out using a suspension of promastigotes in buffered saline solution following the protocol described, in full detail, by the World Organization for Animal Health (OIE, 2008).

\section{Entomological, epidemiological and ecological variables at the household level}

A series of variables, potentially associated with canine cutaneous leishmaniasis pathogen exposure, were quantified for each one of the households (and/or their peridomiciliary environments) enrolled in our study.

Entomological variables. SF were sampled once per month (April-June 2010) using modified light traps with an additional LED light (Chaves et al. 2013). Two traps were set from $6 \mathrm{pm}$ to $6 \mathrm{am}$, at $2 \mathrm{~m}$ height in each household, and at the same site during the three sampling periods. One trap was placed inside the main room of the household (domicile) and the other trap over vegetation within a $50 \mathrm{~m}$ radius from the house (peridomicile). SF were removed from the traps and stored at $-20^{\circ} \mathrm{C}$ to kill the samples, which were subsequently preserved in $70 \%$ alcohol, separated by sex and identified to the species level based on the male genitalia and female spermathecae following the taxonomic key of Young and Duncan (1994). For the analysis, we employed the average from the three monthly observations, in order to have a reliable estimate of SF abundance. A detailed inventory of the collected sand flies species was presented by Calzada et al. (2013).

Epidemiological variables. We recorded the number of humans, the number of humans with active and parasitologically confirmed leishmaniasis lesions, the number of humans with past leishmaniasis lesions in each household. A full and detailed description of epidemiological data collection and patterns of clinical cutaneous leishmaniasis in humans at the study site was presented by Saldaña et al. (2013).

Ecological variables. For each household we estimated: a housing destituteness index, which quantified how different elements of housing construction and materials rendered houses differentially suitable habitats for SF; a peridomicile index, that quantified the abundance and availability of adult SF resting sites in a peridomicile; a vegetation index, that measured natural vegetation vertical structure; the richness, i.e. number of species, of domestic and wildlife animals; 
an index of domestic animal abundance, which weighted the abundance of different domestic species belonging to a household; and an index of wild animal presence, which weighted the commonness of different wildlife species sighted by householders. A detailed description of data collection and the estimation of each index was presented by Chaves et al. (2013).

\section{Ethical approval}

This study was evaluated and approved by the National Review Board, Comité Nacional de Bioética de la Investigación, Instituto Conmemorativo Gorgas de Estudios de la Salud, Ciudad de Panamá, República de Panamá (561/CNBI/ICGES/06), and by ICGES Institutional Animal Care and Use Committee (IACUC, 2006/02). The study was in accordance with law No. 23 of January 151997 (Animal Welfare Assurance) of República de Panamá.

\section{IFAT seropositive assignation}

The samples were considered positive when titers were $\geq 1: 40$ (Dantas-Torres et al. 2010). The positive control was a serum sample from a dog naturally infected with L. Viannia panamensis from central Panama with a 1:160 titer.

\section{ELISA seropositive assignation from the optical densities}

We assigned as seropositive all dogs whose averaged optical density from the ELISA was above the mean plus three standard deviations (S.D.) of the distribution with the smallest mean (i.e. that of the likely seronegative individuals) in a finite mixture model, for details see Supplement S1. This method was chosen because of its robustness to small changes in antibody titres that can emerge from seasonality and/or small variations in laboratory assay performance (Stewart et al. 2009), problems already identified for canine leishmaniasis serodiagnosis (Dye et al. 1993). For the distribution with the smallest mean in the mixture we estimated a mean ( \pm s.E.) of $0 \cdot 102 \pm 0 \cdot 038$, which leads to a seropositivity threshold of $0 \cdot 216$, i.e. any dog whose ELISA optical density was above this value could be considered positive, Fig. S1 shows the distribution of the optical densities from the ELISA test.

\section{Sensitivity and specificity for the IFAT and ELISA as diagnostics of canine cutaneous leishmaniasis caused by Leishmania (Viannia) panamensis}

Sensitivity, the ability of a test to diagnose a true infection, and specificity, the ability of a test to avoid false negative diagnostics is generally assessed in the presence of a 'gold standard', for example, the direct observation of a parasite or its DNA amplification via PCR (Branscum et al. 2005). Nevertheless, canine cutaneous leishmaniasis is a system where a 'gold standard' is likely not plausible, given the limited tissues where positive PCRs could be expected, and observed differences in diagnosis for tissues from the same dog (Reithinger et al. 2000, 2002). In fact, we attempted parasite isolation and tested parasite presence in blood samples using PCR protocols described in (Miranda et al. 2012), all results were negative, probably because of low parasite loads (Padilla et al. 2002) and the low haematogenicity of Leishmania (Viannia) spp. in dogs (Dereure et al. 1994; Reithinger and Davies, 2002). Therefore, we employed the method developed by Dendukuri and Joseph (2001) to estimate the sensitivity and specificity of two serological diagnostic tests in one population, which employs a Bayesian framework for parameter inference, for details see Supplement S2. For the analysis we assumed the following priors: uniform distributions for the covariance of positive and negative tests, an uninformative beta distribution for the real prevalence in the population, beta distributions with mode $=0.90$ and 5 th percentile $=0.70$ for the specificity and sensitivity of the ELISA, a beta distribution with mode $=0.70$ and 5 th percentile $=0.50$ for the IFAT sensitivity, and a beta distribution with mode $=0.80$ and 5 th percentile $=0.60$ for the IFAT specificity. These distributions were based on reported sensitivities for ELISA and IFAT tests for canine cutaneous leishmaniasis, and reflect the observation that ELISA tests tend to outperform IFAT tests in dogs (Supplement S3). Posterior inferences were based on 100000 realizations that followed 10000 burned-in realizations for the Markov Chain Monte Carlo (MCMC) stabilization. MCMC convergence was assessed by running multiple chains with dispersed starting values. Following the recommendations of Branscum et al. (2005), we also performed a parameter sensitivity analysis, described in Supplement S2. The analyses described in this section were implemented in the BUGS statistical software modifying the code developed by Branscum et al. (2005). Since our study area is also endemic for the etiologic agent of Chagas disease, Trypanosoma cruzi, we also assessed the possibility of cross-reaction for Chagas-Leishmania parasites (Padilla et al. 2002; Castro et al. 2007; Gil et al. 2011) by performing a T. cruzi adsorption of Leishmania spp. seropositive sera (Padilla et al. 2002) and by evaluating three different $T$. cruzi tests on samples from each individual dog. We evaluated how likely were the results similar by mere chance through the estimation of Cohen's coefficient of agreement Kappa (Cohen, 1960), between the Leishmania IFAT and ELISA diagnostic tests with a rapid immunochromatographic test for Chagas disease (Reithinger et al. 2010), a T. cruzi ELISA and a $T$. cruzi IFAT (Pineda et al. 2011), and a gold standard 
based on seropositive reactions for T. cruzi by at least two different tests, following WHO recommendation for T. cruzi serological diagnosis in humans (Pineda et al. 2011).

\section{Risk factors associated with cutaneous leishmaniasis seropositivity in dogs}

To estimate the impact of different risk factors on dog seropositivity patterns we investigated the role of several entomological, ecological and epidemiological variables that were common to dogs belonging to a given household in our study area. We also investigated the joint effect of these householdlevel covariates with individually collected information from each dog health condition.

For the analysis at the household level we employed maximum likelihood Binomial Generalized Linear Models (Bin-GLMs) (Faraway, 2006). We first identified the best entomological covariate via an Akaike Information Criterion (AIC) comparison (Faraway, 2004) of models, or their simplifications, that considered one of the following entomological variables: (i) total abundance of sand flies collected; (ii) abundance of domiciliary and peridomiciliary sand flies; (iii) total abundance of Lutzomyia trapidoi and Lu. panamensis, the main dominant vector species in the study area (Calzada et al. 2013) and the whole República de Panamá (Christensen et al. 1983; Dutari and Loaiza, 2014); (iv) abundance of domiciliary and peridomiciliary Lu. trapidoi and Lu. panamensis. We needed to perform this preliminary selection of entomological variables given their lack of independence, i.e. some variables are an additive function of the other variables, a fact that can lead to parameter estimation identifiability (Faraway, 2004). We performed model selection via AIC, i.e. considered both model likelihood and parameter number, because models were not always nested (simpler models not always had a subset of variables from a more complex model), therefore not comparable via likelihood ratio tests (Venables and Ripley, 2002).

Following the selection of the best entomological covariate, we proceeded to incorporate all epidemiological and ecological household level variables already described in the Entomological, Epidemiological and Ecological variables at the household level section. We then selected the best model following a procedure of backward elimination from a full model, which considered the best entomological covariate plus all the ecological and epidemiological variables. All variables whose single elimination decreased the simpler model AIC in relation to a model incorporating such covariates were discarded at once (Venables and Ripley, 2002). Nevertheless, at the final stage of model selection we compared nested models (simpler models having a subset of variables from a more complex model) with non-significant factors, employing likelihood ratio tests if the AIC was not minimized by the simplest model (Venables and Ripley, 2002). Given the spatial nature of the households, we performed a Moran I index test on the residuals from the model selected as best, in order to ensure the spatial independence of the residuals, an assumption for the proper use of Bin-GLMs (Venables and Ripley, 2002).

For the individual based risk factor assessment we employed Logistic Generalized Estimating Equations Models (Log-GEEM) (Venables and Ripley, 2002; Faraway, 2006). We employed LogGEEM given the nature of the data, where dogs belonging to a same household are not independent observations, a fact constraining the use of simpler regression tools (Chaves, 2010). We assumed independence in the correlation structure of the models, given the ability of Log-GEEM to obtain consistent estimates for the fixed effects even when the correlation structure is incorrect (Venables and Ripley, 2002). For the inference we used a sandwich estimator to obtain robust standard errors, since naïve standard errors are appropriate only when the correlation structure is correct (Faraway, 2006). For the identification of significant risk factors for canine cutaneous leishmaniasis, we began our analysis by building a full model that included the best entomological covariate selected for the BinGLMs, all the epidemiological and ecological variables collected at the household level and information on each dog health condition (physical condition, cutaneous lesions, de-worming and ectoparasite presence), whether the dog slept inside the house and demography (i.e. sex and age). This model was simplified using a procedure similar to the one employed for the Bin-GLMs, but based on the quasilikelihood information criterion (Pan, 2001), the GEEM analog to AIC.

For the models we employed seropositivity results based on ELISA when fitting both the Bin-GLMs and Log-GEEMs. We did not perform this analysis for the IFAT results alone, given the low sensitivity of this technique (see Results section). Bin-GLMs and Log-GEEMs were fitted with the statistical package $\mathrm{R}$ version 2.15.3.

\section{Force of infection $(\lambda)$ and basic reproductive number $\left(R_{0}\right)$ estimation}

We estimated the force of infection $(\lambda)$ assuming that seroconversion dynamics in susceptible dogs followed an irreversible autocatalytic process (Anderson and May, 1991), which can be described by the following non-linear partial differential equation:

$$
\frac{\partial S(a, t)}{\partial t}+\frac{\partial S(a, t)}{\partial a}=\lambda(1-S(a, t))
$$


where $S$ is the fraction of seropositive dogs in a dog population that is composed by susceptible and seropositive dogs. At any given time, denoted by $t$, equation (1) can be integrated as function of the age, denoted by $a$, and assuming all dogs are born susceptible to become seropositive following the exposure to canine cutaneous leishmaniasis pathogens, we can obtain the following function for the proportion of seropositive dogs $(S)$ as function of age $(a)$ :

$$
S=1-e^{-\lambda(a)}
$$

We thus employed our data on seroprevalence $(S)$ and age $(a)$ to estimate $(\lambda)$ with equation (2). We specifically employed maximum likelihood methods for parameter estimation (Bolker, 2008). Supplement S4 has the $\mathrm{R}$ code we employed for the maximum likelihood parameter estimation.

To estimate the basic reproduction number, $R_{0}$, we first built a vertical (Southwood, 1978) survival schedule, i.e. a survivorship curve based on the dog population age structure, in order to estimate dog's life expectancy, i.e. the average lifespan (Carey, 2003) of dogs, at Trinidad de Las Minas. Briefly, a vertical survival schedule assumes the age structure of a population to represent the survival schedule of a population at equilibrium and with a pyramidal age structure, i.e. with a larger proportion of younger than older individuals (Southwood, 1978; Krebs, 1998). Because the dog population at Trinidad de Las Minas has a pyramidal structure, and, on average, each household in rural Panama has a couple of dogs (Herrer and Christensen, $1976 b)$ it can be argued that dogs are a stationary population fulfilling the assumptions for the sound estimation of a vertical survival schedule, denoted by $l(a)$, based on the ratios of consecutive age classes:

$$
l(a+1)=\frac{N(a+1)}{N(a)}
$$

In (3) $l(a+1)$ is defined as the probability of surviving from age $a$ to $a+1$ (Carey, 2003). Since individuals at older ages, i.e. 8 or more years, were few, and their abundance did not monotonically decrease, we followed the standard recommendation of smoothing the $l(a)$ curve (Krebs, 1998). For the smoothing we employed the lowess algorithm (Venables and Ripley, 2002):

$$
\tilde{l}(a)=\operatorname{lowess}(l(a))
$$

With the smoothed survival schedule we calculated dog's life expectancy, $e_{0}$, with the following equation (Southwood, 1978; Krebs, 1998):

$$
e_{0}=\sum_{0}^{\infty} \tilde{l}(a)
$$

And with $e_{0}$, the force of infection $(\lambda)$ and the smoothed survival schedule we estimated $R_{0}$ as follows:

$$
R_{0}=\frac{e_{0}}{\sum_{0}^{\infty} e^{-\lambda a \tilde{l}}(a)}
$$

where (6) makes no specific assumptions about the mortality patterns in the dog population. A detailed derivation of equation (6) and its comparison with expressions that made strong assumptions about population mortality are presented in Supplement S5.

\section{RESULTS}

During our census we counted a total of 52 dogs in the 24 households of our study site, 18 were female and 34 were males. Dogs were present in 22 of the 24 surveyed households, with an average ( \pm s.D.) $2 \cdot 12 \pm 1 \cdot 42$ dog per house. Dog abundance per household ranged from 0 to 6 . The average age ( \pm s.D.) of the dogs was $2.07 \pm 2.94$ years, ranging from less than a month (a puppy still weaning) to 13 years. We took blood samples from all dogs except from the female puppy still weaning that was anaemic and had a very poor body condition score. All subsequent results are based on the 51 animals from which blood samples were taken. We found an ACL seroprevalence of $47 \%$ among dogs. About half of the dogs had a poor physical condition (43\%). Almost all dogs had ticks attached (94\%) and slept outside the houses $(92 \%)$. Although skin diseases/conditions were observed in $31 \%$ of the surveyed dogs, none of them presented clinical lesions or scars suggestive of ACL. Thus, no skin scrapings were analysed for direct parasite detection. Cultures and PCR from blood samples were both negative for Leishmania spp. parasites/DNA. A detailed description of the dog population demographic profile, body condition assessment and serodiagnostic test results by age class are presented in Table 1 .

The Bayesian analysis employed to evaluate the diagnostic accuracy of the Leishmania serological tests, showed the ELISA to outperform IFAT, both in sensitivity and specificity (Table 2). The former has a better ability to capture true seropositive reactions (i.e. sensitivity) close to $80 \%$, while for the latter is $51 \%$. Both ELISA and IFAT tests do not fail to detect pathogen free individuals (i.e. specificity) about $80 \%$ of the times. This means that, in general, seropositive patterns detected with ELISA are more likely to be correct (Table S1). A sensitivity analysis for the priors employed in the estimation of sensitivity and specificity of the tests showed the estimates to be robust to assumptions employed in the estimation, nevertheless the sign of the associations between tests outcomes changed (Table S2). However, assumptions about the sign 
Table 1. Demography, health condition and cutaneous leishmaniasis seropositivity in a dog population from Trinidad de Las Minas in Panamá

\begin{tabular}{|c|c|c|c|c|c|c|c|c|}
\hline $\begin{array}{l}\text { Age - } \\
\text { years }\end{array}$ & $\begin{array}{l}\text { Total } \\
\text { (females) }\end{array}$ & $\begin{array}{l}\text { Poor } \\
\text { physical } \\
\text { condition }\end{array}$ & $\begin{array}{l}\text { Ectoparasite } \\
\text { presence }\end{array}$ & $\begin{array}{l}\text { Sleeping } \\
\text { inside the } \\
\text { house }\end{array}$ & $\begin{array}{l}\text { Cutaneous } \\
\text { lessons }\end{array}$ & ELISA+ & IFAT+ & $\begin{array}{l}\% \text { Sero- } \\
\text { prevalence }^{\mathrm{a}}\end{array}$ \\
\hline Below 1 & $12(5)$ & 8 & 12 & 1 & 0 & 4 & 1 & $33(4)$ \\
\hline $1-2$ & $16(6)$ & 6 & 15 & 1 & 7 & 6 & 2 & $38(6)$ \\
\hline $3-4$ & $12(2)$ & 4 & 10 & 1 & 4 & 7 & 5 & $67(8)$ \\
\hline 5 or more & $11(4)$ & 4 & 11 & 1 & 4 & 7 & 7 & $73(8)$ \\
\hline
\end{tabular}

a The value inside parenthesis indicates the number of seropositives obtained by at least one method.

Table 2. Sensitivity and specificity estimates for canine cutaneous leishmaniasis ELISA and IFAT diagnostic tests. 95\% CI indicate the 95\% Bayesian credible intervals

\begin{tabular}{|c|c|c|c|c|}
\hline Diagnostic test & Parameter & Mean & $95 \% \mathrm{CI}$ & \\
\hline \multirow[t]{2}{*}{ ELISA } & Sensitivity & $0 \cdot 79$ & $0 \cdot 67$ & $0 \cdot 90$ \\
\hline & Specificity & $0 \cdot 84$ & $0 \cdot 62$ & 0.97 \\
\hline \multirow[t]{2}{*}{ IFAT } & Sensitivity & $0 \cdot 51$ & $0 \cdot 38$ & 0.63 \\
\hline & Specificity & $0 \cdot 77$ & $0 \cdot 48$ & 0.95 \\
\hline
\end{tabular}

of these associations do not interfere with the proper estimation of sensitivity and specificity, thus further ensuring the soundness of our analysis. Similarly, the multiple Markov chains employed to generate all estimates converged, thus ensuring a valid inferences from our Bayesian Analysis. Further, evidence for the superior quality of the ELISA based diagnosis came from the fact that sera adsorption on T. cruzi antigens did not prevent the ELISA Leishmania reactions suggesting the lack of positive due to crossreactions with $T$. cruzi, and the likely co-exposure L. panamensis and T. cruzi parasites. From 9 positive dogs to T. cruzi according to the gold standard of two T. cruzi diagnostic tests, 7 dogs were ACL seropositive according to the ELISA test and 9 to the IFAT. The two samples diagnosed as Leishmania positive by IFAT and not with ELISA (Table S1) reacted positively with at least two of the Chagas diagnostic tests, thus suggesting a likely cross-reaction. In fact, the Cohen's kappa coefficients of agreement between the Leishmania IFAT and the Chagas tests had values above $0 \cdot 6$ (Table $\mathrm{S} 3$ ), which can be considered as a substantial, if above $80 \%$ excellent, agreement (Landis and Koch, 1977). In contrast, the agreement between the Chagas tests and the Leishmania ELISA was poor (Landis and Koch, 1977), with kappa coefficients below 0.4 in all cases (Table S3). For reference the kappa between the Leishmania ELISA and IFAT was 0.415 (Table S3) which is moderate (Landis and Koch, 1977). Thus, given the low sensitivity and specificity of the IFAT, likely derived from the cross-reaction with
T. cruzi, most subsequent analysis will be based on ELISA results.

The spatial location of the seropositive dogs according to ELISA (Fig. 1A) and IFAT (Fig. 1B) overlapped with the presence of Lutzomyia trapidoi (Fig. 1C) and Lu. panamensis (Fig. 1D), especially with the domiciliary abundance of these two species (Fig. 1). Model selection for risk factors at the household level showed that domiciliary Lu. trapidoi abundance was the main risk factor associated with ELISA (Table S4) L. panamensis seropositive diagnostics in a household, with odds for ELISA seropositive diagnosis increasing 2.28 times by each Lu. trapidoi sand fly caught by night trap of sand-fly sampling ('Table 3). The Moran's I test supports the lack of spatial autocorrelation in residuals from the binomial generalized linear models we fitted in Table 3 , a fact that, in addition to the confirmation of generalized linear model assumptions about the error, suggests our inferences are sound. The analysis from data gathered for the individual dogs only included sex, age, skin lesion presence, deworming and physical condition. We excluded tick presence and sleeping outside the house given their low variability. Model selection showed that for ELISA (Table S5) only domiciliary Lu. trapidoi abundance by trap-night and age were significant risk factors. In the case of ELISA the odds of seropositive reaction in a given $\mathrm{dog}$ increased $3 \cdot 39$ times and 1.35 times, respectively, by each sand fly caught inside the house where a dog belonged and dog age in years (Table 4 ). 

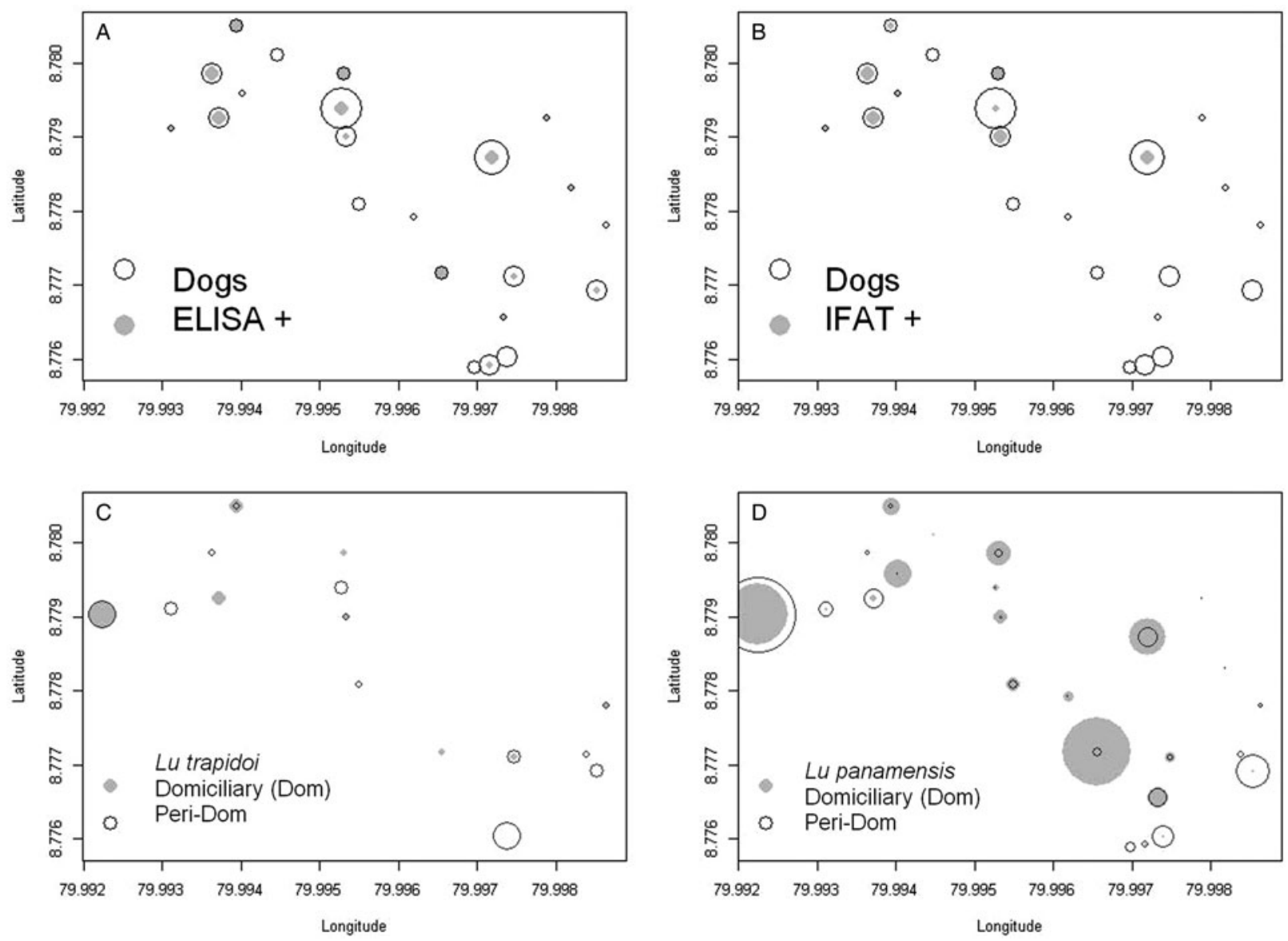

Fig. 1. Seroprevalence and dominant vector species abundance. In all panels symbol size is proportional to abundance. (A) ELISA, circles are proportional to the number of dogs (Dogs) and grey dots to the number of ELISA seropositive dogs (ELISA+), symbol size in the inset legend corresponds to two individuals. (B) Indirect Immunofluorescence test, IFAT, circles are proportional to the number of dogs (Dogs) and grey dots to the number of IFAT seropositive dogs (IFAT + ), symbol size in the inset legend corresponds to two individuals. (C) Lutzomyia trapidoi abundance, for symbol interpretation please refer to the inset legend, where symbol size corresponds to two individuals. (D) Lu. panamensis abundance, for symbol interpretation please refer to the inset legend, where symbol size corresponds to four individuals in the domiciliary environment and 20 individuals in the peridomiciliary environment. In the $y$ - and $x$-axis $0 \cdot 001$ degree of latitude/longitude are approximately $110 \mathrm{~m}$.

Table 3. Parameter estimates for the best binomial generalized linear models explaining the odds ratio of cutaneous leishmaniasis ELISA seropositive reactions in dogs at the household level. 95\% CI indicate the 95\% maximum likelihood confidence intervals for the estimated odds

\begin{tabular}{llrl}
\hline \hline Parameter & Odds ratio (95\% CI) & Estimate $( \pm$ s.E.) & $P$ \\
\hline Intercept & 1 & - & - \\
Lutzomia trapidoi abundance inside the houses & $2 \cdot 28(1 \cdot 07-7 \cdot 13)$ & $0 \cdot 948( \pm 0 \cdot 473)$ & $0 \cdot 033 *$ \\
Moran's I test for spatial autocorrelation in the residuals & - & -0.085 & $0 \cdot 568$ \\
\hline \hline
\end{tabular}

* Statistically significant $(P<0 \cdot 05)$.

The vertical life table we built based on the dog population age profile, estimated dog age expectation at birth $\left(e_{0}\right)$ to be close to three and a half years (Fig. 2). Figure 3 shows the estimated age specific seroprevalence curves, force of infection $(\lambda)$ and basic reproductive number $\left(R_{0}\right)$ with ELISA. $R_{0}$ was significantly larger than 1 , indicating that the disease was endemically established in dogs when our survey was done and that, on average, new infections will keep appearing in a fully susceptible dog population, ceteris paribus (i.e. everything else being equal). Finally, $R_{0}$ estimates (Fig. 3) by equation (6) highlight how estimates based on the 'extreme' assumptions of low mortality to the age expectation at birth or constant age independent mortality are likely biased (Table S6), making 
Table 4. Parameter estimates for the best logistic generalized estimating equation models explaining cutaneous leishmaniasis seropositivity by ELISA in dogs. Houses were considered as the clustering factor in the analysis

\begin{tabular}{llll}
\hline \hline Parameter & Odds ratio & Estimate $( \pm$ sandwich s.E.) & $Z$ \\
\hline Intercept & 1 & - & - \\
Lutzomia trapidoi abundance inside the houses & $3 \cdot 39$ & $1 \cdot 22( \pm 0 \cdot 39)$ & $3 \cdot 122^{*}$ \\
Dog age & $1 \cdot 35$ & $0 \cdot 30( \pm 0 \cdot 09)$ & $3 \cdot 395^{*}$ \\
\hline \hline
\end{tabular}

* Statistically significant $(P<0 \cdot 05)$.

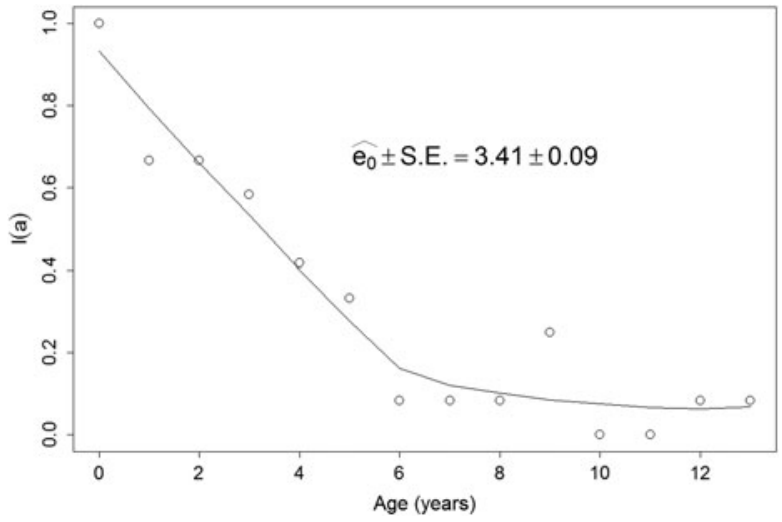

Fig. 2. Age specific survival $(l(a))$ schedule from a vertical life table. Open circles are the raw estimates from the data. The solid line represents a lowess smoothed survival schedule. Life expectancy $\left(e_{0}\right)$ was estimated with the lowess smoothed survival $l(a)$ curve and equation (5).

preferable the use of the population survival schedule when available.

\section{DISCUSSION}

As a zoonosis ACL is particularly interesting given the diversity of hosts that can be infected by NWC Leishmania spp. parasites (Garnham, 1965; Ashford, 1997). Not all host species are reservoirs of infection, some species are incidental hosts, i.e. dead-end hosts from which the parasites would unlikely infect vectors, while, by contrast, other species are true reservoirs, with a dynamic feedback in the transmission between sand fly vectors and the vertebrate hosts (Chaves and Hernandez, 2004; Chaves et al. 2007, 2008b). The incrimination of reservoir hosts in ACL transmission critically depends on high quality diagnostics, which over recent years have significantly evolved. Nevertheless, the absence of a gold standard has remained a major challenge (Reithinger et al. 2000; Reithinger and Davies, 2002), which nowadays can be partially circumvented due to the development of Bayesian statistical tools, that allow the estimation of sensitivity and specificity in the absence of a gold standard (Branscum et al. 2005). In our study, we benefited from these 'new' Bayesian techniques and were able to confirm that a L. panamensis ELISA was a

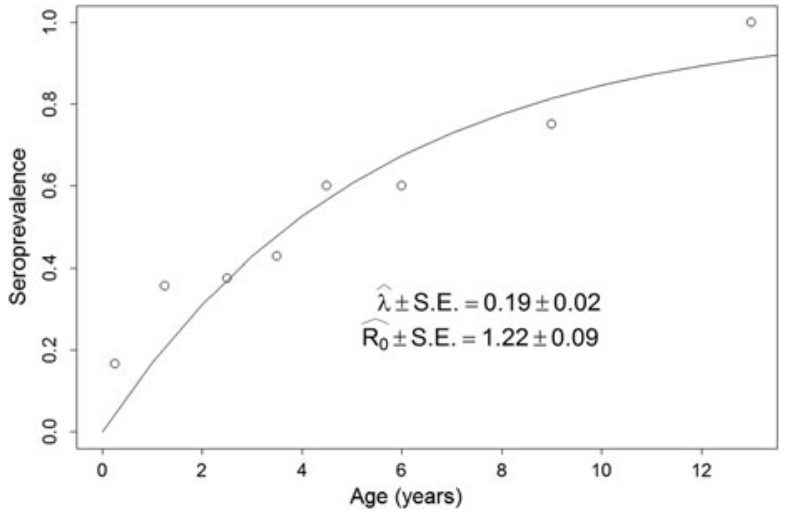

Fig. 3. Force of infection $(\lambda)$ and basic reproduction number $\left(R_{0}\right)$ estimates. Estimates are based on the age specific seroprevalence from ELISA. $\lambda$ was estimated via the maximum likelihood fitting of equation (2) to the seroprevalence data (open circles). For a full description of the maximum likelihood procedure see Supplement S4. $R_{0}$ was estimated with equation (6).

diagnostic tool able to outperform the commonly employed IFAT, an observation in accordance with several previous studies (Reithinger and Davies, 1999; Castro et al. 2007). Nevertheless, we consider extremely desirable the development of even more sensitive and specific diagnostic tests. For example, rapid diagnostic tests like the rK39 RDT recently developed for canine visceral leishmaniasis (Quinnell et al. 2013) can significantly improve studies evaluating the reservoir role of dogs across the distinct ACL eco-epidemiological settings. However, the potential of recombinant antigens and/or rapid tests for Leishmania Viannia spp. serological diagnosis has not been validated yet.

The performance (sensitivity and specificity) of serological diagnostics methods are influenced significantly by the characteristics of the antigen used. In this study we used L. panamensis promastigotes (IFAT) or their soluble crude extracts (ELISA) following procedures previously described and recommended by the World Organization for Animal Health. Our analysis showed that IFAT was more likely to cross-react with $T$. cruzi, which is a problem for proper diagnosis in areas where ACL and Chagas disease co-occur (Padilla et al. 2002), further reinforcing the use of ELISA-like and 
other sensitive and specific diagnostics. New advances for the identification of Leishmania species with polymorphism specific PCR (Marco et al. 2012) can also greatly improve the understanding of circulating parasite species in endemic areas. In that sense, our study was limited given that we can only assume that the circulating species was $L$. panamensis, the species we were able to identify in biopsies and skin scrapings from humans with active skin lesions living in the studied area (Saldaña et al. 2013).

Results about risk factors for the exposure to NWC Leishmania spp. were within what could be expected. Seropositive reactions, indicative of exposure to NWC Leishmania spp. parasites, were more likely to occur in houses with Lu. trapidoi, and further increased with the abundance of this dominant vector species, the main ACL vector in the Republic of Panamá (Christensen et al. 1983; Dutari and Loaiza, 2014) and one of the dominant vector species at our study site (Calzada et al. 2013; Chaves et al. 2013; Saldaña et al. 2013). The skin lesions we observed in dogs were not typical of ACL. This might reflect that in tropical regions, like our study site, skin diseases in dogs due to ectoparasites, bacteria and fungus are very frequent and may result in similar skin lesions, complicating the clinical diagnosis of ACL (Fung et al. 2014).

Also, the exposure risk increased with domiciliary sand fly abundance, probably reflecting the fact that dogs sleep just outside the households, a place where sand fly density is likely more closely associated with the intra-domiciliary sand fly abundance than with the peridomiciliary abundance sampled by a trap within $50 \mathrm{~m}$ from the household, especially in light of the poor dispersal ability of sand flies, which occurs mainly within $20 \mathrm{~m}$ (Alexander, 1987; Morrison et al. 1993). Interestingly, previous studies in Panama observed that Lu. trapidoi was attracted to dogs, humans, but also to wildlife species that could act as NWC Leishmania spp. reservoirs (Christensen and Herrer, 1973; Herrer and Christensen, $1976 a, b$ ). In our study site, the risk for clinical human ACL increased with the abundance of Lu. gomezi and Lu. trapidoi (Saldaña et al. 2013), both NWC Leishmania spp. competent sand fly vectors (Dutari and Loaiza, 2014). These observations deserve to be placed in light of our previous findings, since trends of sand fly infestation at our study site are closely related with housing quality. Destitute houses were more likely to be reinfested by sand flies after vector control (Chaves et al. 2013), thus highlighting that domestic dog exposure to NWC Leishmania spp. parasites might be linked with the poverty context of ACL transmission. Exposure also increased with age, probably reflecting the accumulation of infective bites through the lifespan of a dog in an endemic area (Quinnell et al. 1997), a pattern observed in endemic areas for other vector-borne diseases (Anderson and May, 1991).

Our model to study age specific canine ACL seroprevalence patterns had two major differences with a previous study (Reithinger et al. 2003a). To the best of our knowledge, that study has been the only attempt to model and estimate the force of infection and $R_{0}$ of canine ACL in dogs. First, we did not consider the possibility of recovery in dogs, i.e. that seropositive dogs could become seronegative, which is a limitation of cross-sectional studies like ours that cannot measure seronegative conversions. Nevertheless, Reithinger et al. (2003a) present parameters that were not significantly different from 0 , suggesting that likelihood of recovery is very small, and that temporal variability in the samples was not properly accounted when assigning ACL seropositivity to dogs. Observations by Marco et al. (2001) indeed suggest that negative seroconversion is highly unlikely, and Aguilar et al. (1989) found that some temporal variability might exist leading to inconsistent serodiagnosis in cohort studies, suggesting that ACL exposure leads to long-lasting seroconversion. In addition, this might happen when transmission is endemic and exposure is sustained. In that sense we want to highlight that we used a cutting edge method to assign seropositive status to our ELISA samples (Bretscher et al. 2013) and that, for the purpose of an abstraction (Levins, 1968, 2006), not considering dog recovery, given that dog mortality is likely a more important process regulating transmission in dogs, does not invalidate our model. Second, we did not consider dog mortality as a constant, nor did we use models assuming that mortality was constant across ages (Quinnell et al. 1997; Reithinger et al. 2003a). The landmark work by Anderson and May (1991), derived results assuming a constant mortality given the elegance of closed form solutions that can be expressed with simple formulas. Nevertheless, in no instance Anderson and May (1991) reified this to be the case in nature. Our data clearly showed that estimates using the survival schedule can produce estimates (Fig. 3) contained between the extreme cases derived by Anderson and May (1991) (Table S6). Thus, our estimates are likely more accurate and can more realistically account for the non-constant nature of mortality observed across organisms (Carey, 2001, 2003). Similarly, although there were no significant differences between the $R_{0}$ estimates based on ELISA and ELISA or IFAT diagnostics, the poor specificity, and potential cross-reactivity with $T$. cruzi, of the IFAT highlights how the pooling of results by different diagnostic tests (Reithinger et al. 2003a) may not be warranted, given underlying differences in data quality (Boggild et al. 2010).

Age specific seroprevalence patterns suggest that canine ACL was endemically established at Trinidad 
de Las Minas when our cross-sectional study took place. $R_{0}$, the basic reproduction number, was significantly above 1 , meaning that exposure of dogs to NWC Leishmania spp. parasites was endemically established (Chaves and Hernandez, 2004). Here, we want to stress that endemic establishment does not imply that dogs are reservoirs, since as long as the disease is endemically established in the true reservoirs (Chaves et al. 2008b), cutaneous leishmaniasis can be expected to be established in any incidental host that gets into frequent contact with infected sand flies (Chaves et al. 2007). Here, it is also important to bring results from previous studies in Panamá, where vectors associated with human transmission do not often feed in dogs (Telford Jr et al. 1972; Herrer and Christensen, 1976a, $b$; Christensen and Herrer, 1980), unlike the two toed sloth, which showed a dynamical feedback of transmission with sand fly vectors, with a high success probability of vector infection (Thatcher and Hertig, 1966; Christensen and Herrer, 1972, 1979; Herrer and Christensen, 1980) significantly higher than what has been observed in dogs (Hernández et al. 2006; Travi et al. 2006) or humans (Rojas and Scorza, 1990). In fact, we observed two toed sloths in our study area, from which we were able to isolate L.panamensis parasites (González et al. 2015), a competent reservoir from which L.panamensis has been previously isolated (Herrer and Christensen, 1980). In summary, the presence of alternative likely reservoirs hosts, the relative low attraction of sand fly vectors to dogs when compared with sloths, the low probability of sand fly infection on dogs, as well as the low haematogeneicity of NWC Leishmania spp. parasites (Pirmez et al. 1988; Reithinger et al. 2000, 2002), low parasite density that has been detected in blood (Padilla et al. 2002) and given that most ACL in Panamá is due to infections with $L$. panamensis (Chaves et al. 2014) we think that dogs are not a significant reservoir in the L. panamensis ACL epidemiology in our study site, which is likely illustrative of ACL epidemiology in the whole Republic of Panamá.

\section{SUPPLEMENTARY MATERIAL}

To view supplementary material for this article, please visit http://dx.doi.org/10.1017/S0031182015000475.

\section{ACKNOWLEDGEMENTS}

Ms Junko Sakemoto offered valuable administrative support at Nagasaki University.

\section{FINANCIAL SUPPORT}

This study was funded by a Gorgas Memorial Research Award of the American Society of Tropical Medicine and Hygiene to LFC, NETROPICA grant $04-\mathrm{N}-2010$ to JEC and LFC, SENACYT grant CCP06-040 to JEC and AS, and Nagasaki University (Program for Nurturing Global Leaders in Tropical and Emerging Communicable Diseases) to LFC.

\section{REFERENCES}

Aguilar, C. M., Rangel, E. F., Garcia, L., Fernandez, E., Momen, H., Grimaldi Filho, G. and Vargas, Z. d. (1989). Zoonotic cutaneous leishmaniasis due to Leishmania (Viannia) braziliensis associated with domestic animals in Venezuela and Brazil. Memorias Do Instituto Oswaldo Cruz 84, 19-28.

Alexander, B., Agudelo, L. A., Navarro, F., Ruiz, F., Molina, J., Aguilera, G. and Quiñones, M. L. (2001). Phlebotomine sandflies and leishmaniasis risks in Colombian coffee plantations under two systems of cultivation. Medical and Veterinary Entomology 15, 364-373.

Alexander, J. B. (1987). Dispersal of phlebotomine sand flies (Diptera: Psychodidae) in a Colombian coffee plantation. Fournal of Medical Entomology 24, 552-558.

Alvar, J., Vélez, I. D., Bern, C., Herrero, M., Desjeux, P., Cano, J., Jannin, J., Boer, M. d. and the, W. H. O. L. C. T. (2012). Leishmaniasis worldwide and global estimates of its incidence. PLoS ONE 7, e35671

Anderson, R. M. and May, R. M. (1991). Infectious Diseases of Humans: Dynamics and Control. Oxford University Press, Oxford.

Aragão, H. d. B. (1927). Leishmaniose tegumentar e sua transmissão pelos phlebotomos. Memorias Do Instituto Oswaldo Cruz 20, 177-195.

Ashford, R. W. (1997). The leishmaniases as model zoonoses. Annals of Tropical Medicine and Parasitology 91, 693-701.

Boggild, A. K., Ramos, A.P., Espinosa, D., Valencia, B. M., Veland, N., Miranda-Verastegui, C., Arevalo, J., Low, D.E. and Llanos-Cuentas, A. (2010). Clinical and demographic stratification of test performance: a pooled analysis of five laboratory diagnostic methods for American Cutaneous Leishmaniasis. The American fournal of Tropical Medicine and Hygiene 83, 345-350.

Bolker, B. M. (2008). Ecological Models and Data in R. Princeton University Press, Princeton.

Branscum, A. J., Gardner, I. A. and Johnson, W. O. (2005). Estimation of diagnostic-test sensitivity and specificity through Bayesian modeling. Preventive Veterinary Medicine 68, 145-163.

Bretscher, M., Supargiyono, S., Wijayanti, M., Nugraheni, D., Widyastuti, A., Lobo, N., Hawley, W., Cook, J. and Drakeley, C. (2013). Measurement of Plasmodium falciparum transmission intensity using serological cohort data from Indonesian schoolchildren. Malaria fournal 12, 21.

Calzada, J. E., Saldaña, A., Rigg, C., Valderrama, A., Romero, L. and Chaves, L.F. (2013). Changes in phlebotomine sand fly species composition following insecticide thermal fogging in a rural setting of Western Panamá. PLoS ONE 8, e53289.

Carey, J. R. (2001). Insect biodemography. Annual Review of Entomology 46, 79-110.

Carey, J. R. (2003). Longevity: the Biology and Demography of Life Span. Princeton University Press, Princeton, NJ, USA.

Castro, E. A., Thomaz-Soccol, V., Augur, C. and Luz, E. (2007) Leishmania (Viannia) braziliensis: epidemiology of canine cutaneous leishmaniasis in the State of Paraná (Brazil). Experimental Parasitology 117, 13-21. Chaves, L.F., Calzada, J.E., Rigg, C., Valderrama, A., Gottdenker, N. L. and Saldaña, A. (2013). Leishmaniasis sand fly vector density reduction is less marked in destitute housing after insecticide thermal fogging. Parasites \& Vectors 6, 164.

Chaves, L.F. (2010). An entomologist guide to demystify pseudoreplication: data analysis of field studies with design constraints. Fournal of Medical Entomology 47, 291-298.

Chaves, L. F. and Hernandez, M.-J. (2004). Mathematical modelling of American Cutaneous Leishmaniasis: incidental hosts and threshold conditions for infection persistence. Acta Tropica 92, 245-252.

Chaves, L.F., Hernandez, M.-J., Dobson, A.P. and Pascual, M. (2007). Sources and sinks: revisiting the criteria for identifying reservoirs for American cutaneous leishmaniasis. Trends in Parasitology 23, 311-316. Chaves, L. F., Cohen, J. M., Pascual, M. and Wilson, M. L. (2008a). Social exclusion modifies climate and deforestation impacts on a vectorborne disease. PLoS Neglected Tropical Diseases 2, e176.

Chaves, L. F., Hernandez, M.-J. and Ramos, S. (2008b). Simulación de modelos matemáticos como herramienta para el estudio de los reservorios de la Leishmaniasis Cutánea Americana. Divulgaciones matemáticas 16, $125-154$. 
Chaves, L. F., Calzada, J. E., Valderama, A. and Saldaña, A. (2014). Cutaneous Leishmaniasis and Sand Fly fluctuations are associated with El Niño in Panamá. PLoS Neglected Tropical Diseases 8, e3210.

Christensen, H. A. and de Vasquez, A. M. (1982). The tree-buttress biotope: a pathobiocenose of Leishmania braziliensis. The American Fournal of Tropical Medicine and Hygiene 31, 243-251.

Christensen, H. A. and Herrer, A. (1972). Detection of Leishmania braziliensis by xenodiagnosis. Transactions of the Royal Society of Tropical Medicine and Hygiene 66, 798-799.

Christensen, H. A. and Herrer, A. (1973). Attractiveness of sentinel animals to vectors of leishmaniasis in Panama. The American fournal of Tropical Medicine and Hygiene 22, 578-584.

Christensen, H. A. and Herrer, A. (1979). Susceptibility of Sand Flies (Diptera: Psychodidae) to trypanosomatidae from two-toed sloths (Edentata:Bradypodidae). Fournal of Medical Entomology 16, 424-427.

Christensen, H. A. and Herrer, A. (1980). Panamanian Lutzomyia (Diptera: Psychodidae) host attraction profiles. Fournal of Medical Entomology 17, 522-528.

Christensen, H. A., Fairchild, G. B., Herrer, A., Johnson, C. M., Young, D. G. and Vasquez, A. M. d. (1983). The ecology of cutaneous leishmaniasis in the republic of Panama. Fournal of Medical Entomology 20, 463-484.

Cohen, J. (1960). A coefficient of agreement for nominal scales. Educational and Psychological Measurement 20, 37-46.

Colombo, F., Odorizzi, R.F. N., Laurenti, M., Galati, E. B., Canavez, F. and Pereira-Chioccola, V. (2011). Detection of Leishmania (Leishmania) infantum RNA in fleas and ticks collected from naturally infected dogs. Parasitology Research 109, 267-274.

Dantas-Torres, F. (2007). The role of dogs as reservoirs of Leishmania parasites, with emphasis on Leishmania (Leishmania) infantum and Leishmania (Viannia) braziliensis. Veterinary Parasitology 149, 139-146. Dantas-Torres, F., de Paiva-Cavalcanti, M., Figueredo, L. A., Melo, M.F., da Silva, F. J., da Silva, A. L., Almeida, E. L. and Brandão-Filho, S. P. (2010). Cutaneous and visceral leishmaniosis in dogs from a rural community in northeastern Brazil. Veterinary Parasitology 170, 313-317.

Dendukuri, N. and Joseph, L. (2001). Bayesian approaches to modeling the conditional dependence between multiple diagnostic tests. Biometrics 57, 158-167.

Dereure, J., Espinel, I., Barrera, C., Guerrini, F., Martini, A., Echevarria, R., Guderian, R. H. and Le Pont, F. (1994). Leishmaniose en Equateur. 4. Infestation naturelle du chien par Leishmania panamensis. Annales de la Societe belge de Medicine tropicale 74, 29-33.

Dutari, L. and Loaiza, J. (2014). American cutaneous leishmaniasis in Panama: a historical review of entomological studies on anthropophilic Lutzomyia sand fly species. Parasites E Vectors 7, 218

Dye, C., Vidor, E. and Dereure, J. (1993). Serological diagnosis of leishmaniasis: on detecting infection as well as disease. Epidemiology \& Infection 110, 647-656.

Falqueto, A., Sessa, P. A., Varejão, J. B. M., Barros, G. C., Momen, H. and Grimaldi Júnior, G. (1991). Leishmaniasis due to Leishmania braziliensis in Espírito Santo state, Brazil: further evidence on the role of dogs as a reservoir of infection for humans. Memorias Do Instituto Oswaldo Cruz 86, 499-500.

Faraway, J. J. (2004). Linear Models with R. CRC Press, Boca Raton.

Faraway, J. J. (2006). Extending the Linear Model with R: Generalized Linear, Mixed Effects and Nonparametric Regression Models. CRC Press, Boca Raton.

Fung, H. L., Calzada, J., Saldaña, A., Santamaria, A. M., Pineda, V., Gonzalez, K., Chaves, L. F., Garner, B. and Gottdenker, N. (2014). Domestic dog health worsens with socio-economic deprivation of their home communities. Acta Tropica 135, 67-74

Garcia, L., Kindt, A., Bermudez, H., Llanos-Cuentas, A., De Doncker, S., Arevalo, J., Quispe Tintaya, K. W. and Dujardin, J.-C. (2004). Culture-independent species typing of neotropical Leishmania for clinical validation of a PCR-based assay targeting heat shock protein 70 genes. Fournal of Clinical Microbiology 42, 2294-2297.

Garnham, P. C. C. (1965). The Leishmanias, with special reference to the role of animal reservoirs. American Zoologist 5, 141-151.

Gil, J.F., Hoyos, C. L., Cimino, R. O., Krolewiecki, A. J., Lopez Quiroga, I., Cajal, S.P., Juarez, M., Garcia Bustos, M.F., Mora, M. C., Marco, J.D. and Nasser, J. R. (2011). Role of three ELISA tests using promastigote homogenates of Leishmania braziliensis, L. amazonensis and L. guyanensis in the diagnosis of tegumentary leishmaniasis. Medicina 71, 420-428.

González, K., Calzada, J. E., Saldaña, A., Rigg, C., Alvarado, G., Rodríguez-Herrera, B., Kitron, U. D., Adler, G. H., Gottdenker, N.
L., Chaves, L.F. and Baldi, M. (2015). Survey of wild mammal hosts of Cutaneous Leishmaniasis parasites in Panamá and Costa Rica. Tropical Medicine and Health 43, 75-78.

Hernández, D., Rojas, E., Scorza, J. V. and Jorquera, A. (2006). Infectividad del perro (Canis familiaris) para Lutzomyia youngi en Trujillo, Venezuela. Biomedica 26, s242-s248.

Herrer, A. and Christensen, H. A. (1976a). Epidemiological patterns of Cutaneous Leishmaniasis in Panama: III. Endemic persistence of the disease. The American Fournal of Tropical Medicine and Hygiene 25, 54-58.

Herrer, A. and Christensen, H. A. (1976b). Natural Cutaneous Leishmaniasis among dogs in Panama. The American fournal of Tropical Medicine and Hygiene 25, 59-63.

Herrer, A. and Christensen, H. A. (1980). Leishmania braziliensis in the Panamanian two-toed sloth, Choloepus hoffmanni. The American fournal of Tropical Medicine and Hygiene 29, 1196-1200.

Krebs, C. J. (1998). Ecological Methodology. New York: Benjamin Cummings.

Landis, J. R. and Koch, G. G. (1977). The measurement of observer agreement for categorical data. Biometrics 33, 159-174.

Lemos, E. M., Laurenti, M. D., Moreira, M. A. B., Reis, A. B., Giunchetti, R. C., Raychaudhuri, S. and Dietze, R. (2008). Canine visceral leishmaniasis: performance of a rapid diagnostic test (Kalazar Detect $^{\mathrm{TM}}$ ) in dogs with and without signs of the disease. Acta Tropica 107, 205-207.

Levins, R. (1968). Evolution in Changing Environments. Some Theoretical Explorations. Princeton University Press, Princeton.

Levins, R. (2006). Strategies of abstraction. Biology \& Philosophy 21, $741-755$.

Marco, J., Barroso, P., Mimori, T., Locatelli, F., Tomatani, A., Mora, M., Cajal, S., Nasser, J., Parada, L., Taniguchi, T., Korenaga, M., Basombrio, M. and Hashiguchi, Y. (2012). Polymorphism-specific PCR enhances the diagnostic performance of American tegumentary leishmaniasis and allows the rapid identification of Leishmania species from Argentina. BMC Infectious Diseases 12, 191.

Marco, J.D., Padilla, A. M., Diosque, P., Fernández, M. M., Malchiodi, E. L. and Basombrío, M. A. (2001). Force of infection and evolution of lesions of canine tegumentary leishmaniasis in Northwestern Argentina. Memorias Do Instituto Oswaldo Cruz 96, 649-652.

Miranda, A., Saldaña, A., González, K., Paz, H., Santamaría, G., Samudio, F. and Calzada, J. E. (2012). Evaluation of PCR for cutaneous leishmaniasis diagnosis and species identification using filter paper samples in Panama, Central America. Transactions of the Royal Society of Tropical Medicine and Hygiene 106, 544-548.

Morrison, A. C., Ferro, C., Morales, A., Tesh, R. B. and Wilson, M. L. (1993). Dispersal of the sand fly Lutzomyia longipalpis (Diptera: Psychodidae) at an endemic focus of Visceral Leishmaniasis in Colombia. Fournal of Medical Entomology 30, 427-435.

OIE (2008). Manual of Diagnostic Tests and Vaccines for Terrestrial Animals, 6th Edn. Office International de Epizooties, Paris, France.

Padilla, A. M., Marco, J. D., Diosque, P., Segura, M. A., Mora, M. C., Fernández, M. M., Malchiodi, E. L. and Basombrío, M. A. (2002). Canine infection and the possible role of dogs in the transmission of American tegumentary leishmaniosis in Salta, Argentina. Veterinary Parasitology 110, 1-10.

Pan, W. (2001). Akaike's information criterion in generalized estimating equations. Biometrics 57, 120-125.

Pedroso, A. M. (1913). Leishmania local de cão. Annais Paulistas de Medicina e Cirurgia 1, 33-40.

Pineda, V., Saldaña, A., Monfante, I., Santamaría, A. Gottdenker, N. L., Yabsley, M. J., Rapoport, G. and Calzada, J.E. (2011). Prevalence of trypanosome infections in dogs from Chagas disease endemic regions in Panama, Central America. Veterinary Parasitology 178, 360-363.

Pirmez, C., Coutinho, S. G., Marzochi, M. C. A., Nunes, M. P. and Grimaldi, G. (1988). Canine American cutaneous leishmaniasis: a clinical and immunological study in dogs naturally infected with Leishmania braziliensis braziliensis in an endemic area of Rio de Janeiro, Brazil. The American fournal of Tropical Medicine and Hygiene 38, 52-58. Quinnell, R. J., Courtenay, O., Garcez, L. and Dye, C. (1997). The epidemiology of canine leishmaniasis: transmission rates estimated from a cohort study in Amazonian Brazil. Parasitology 115, 143-156.

Quinnell, R. J., Carson, C., Reithinger, R., Garcez, L. M. and Courtenay, O. (2013). Evaluation of rK39 rapid diagnostic tests for Canine Visceral Leishmaniasis: longitudinal study and meta-analysis. PLoS Neglected Tropical Diseases 7, e1992.

Quintana, M. G., Salomon, O. D., De Grosso, M. and Lizarralde, S. (2010). Distribution of Phlebotomine Sand Flies (Diptera: Psychodidae) 
in a Primary Forest-Crop Interface, Salta, Argentina. Fournal of Medical Entomology 47, 1003-1010.

Reithinger, R. and Davies, C. R. (1999). Is the domestic dog (Canis familiaris) a reservoir host of American cutaneous leishmaniasis? A critical review of the current evidence. The American Fournal of Tropical Medicine and Hygiene 61, 530-541.

Reithinger, R. and Davies, C. R. (2002). American cutaneous leishmaniasis in domestic dogs: an example of the use of the polymerase chain reaction for mass screening in epidemiological studies. Transactions of the Royal Society of Tropical Medicine and Hygiene 96, S1/123-S121/126.

Reithinger, R., Lambson, B. E., Barker, D. C. and Davies, C. R. (2000). Use of PCR to detect Leishmania (Viannia) spp. in dog blood and bone marrow. Fournal of Clinical Microbiology 38, 748-751.

Reithinger, R., Lambson, B. E., Barker, D. C., Counihan, H., Espinoza, J. C., González, J. S. and Davies, C. R. (2002). Leishmania (Viannia) spp. dissemination and tissue tropism in naturally infected dogs (Canis familiaris). Transactions of the Royal Society of Tropical Medicine and Hygiene 96, 76-78.

Reithinger, R., Espinoza, J. C. and Davies, C. R. (2003a). The transmission dynamics of canine american cutaneous Leishmaniasis in Huánuco, Perú. The American Fournal of Tropical Medicine and Hygiene 69, 473-480. Reithinger, R., Espinoza, J. C., Llanos-Cuentas, A. and Davies, C. R. (2003b). Domestic dog ownership: a risk factor for human infection with Leishmania (Viannia) species. Transactions of the Royal Society of Tropical Medicine and Hygiene 97, 141-145.

Reithinger, R., Grijalva, M. J., Chiriboga, R. F., Alarcón de Noya, B., Torres, J. R., Pavia-Ruz, N., Manrique-Saide, P., Cardinal, M. V. and Gürtler, R.E. (2010). Rapid detection of Trypanosoma cruzi in human serum by use of an immunochromatographic dipstick test. Fournal of Clinical Microbiology 48, 3003-3007.

Rojas, E. and Scorza, J. V. (1990). Xenodiagnóstico con Lutzomyia youngi en casos Venezolanos de leishmaniasis cutánea por Leishmania braziliensis. Memorias Do Instituto Oswaldo Cruz 84, 29-34.

Saldaña, A., Chaves, L.F., Rigg, C. A., Wald, C., Smucker, J. E. and Calzada, J. E. (2013). Clinical Cutaneous Leishmaniasis rates are associated with household Lutzomyia gomezi, Lu. panamensis, and Lu. trapidoi abundance in Trinidad de Las Minas, Western Panama. The American Fournal of Tropical Medicine and Hygiene 88, 572-574.

Sokal, R. R. and Rohlf, F. J. (1994). Biometry: the Principles and Practices of Statistics in Biological Research, 3rd Edn. W. H. Freeman, New York, USA. Southwood, T.R.E. (1978). Ecological Methods. Chapman \& Hall, London.

Stewart, L., Gosling, R., Griffin, J., Gesase, S., Campo, J., Hashim, R., Masika, P., Mosha, J., Bousema, T., Shekalaghe, S., Cook, J., Corran, P., Ghani, A., Riley, E. M. and Drakeley, C. (2009). Rapid assessment of malaria transmission using age-specific seroconversion rates. PLoS ONE 4, e6083.

Telford, S. R., Jr., Herrer, A. and Christensen, H. A. (1972). Enzootic cutaneous leishmaniasis in eastern Panama. 3. Ecological factors relating to the mammalian hosts. Annals of Tropical Medicine and Parasitology 66 , 173-179.

Thatcher, V.E. and Hertig, M. (1966). Field studies on the feeding habits and diurnal shelters of some phlebotomus sandflies (Diptera: Psychodidae) in Panama. Annals of the Entomological Society of America 59, 46-52.

Travi, B. L., Tabares, C. J. and Cadena, H. (2006). Leishmania (Viannia) braziliensis infection in two Colombian dogs: a note on infectivity for sand flies and response to treatment. Biomedica 26, s249-s253.

Venables, W. N. and Ripley, B. D. (2002). Modern Applied Statistics with $S$. Springer, New York, USA.

Vergel, C., Walker, J. and Saravia, N. G. (2005). Amplification of human DNA by primers targeted to Leishmania kinestoplast DNA and Post-Genome considerations in the detection of parasites by a polymerase chain reaction. The American fournal of Tropical Medicine and Hygiene 72, 423-429.

Viana, M., Mancy, R., Biek, R., Cleaveland, S., Cross, P. C., LloydSmith, J. O. and Haydon, D. T. (2014). Assembling evidence for identifying reservoirs of infection. Trends in Ecology \& Evolution 29, 270-279.

Young, D. G. and Duncan, M. A. (1994). Guide to the Identification and Geographic Distribution of Lutzomyia Sand Flies in Mexico, the West Indies, Central and South America (Diptera: Psychodidae). Associated Publishers, Gainesville, FL, USA. 